\title{
Kounis syndrome: An additional etiologic factor of myocardial infarction with non-obstructive coronary arteries
}

\author{
Nicholas G. Kounis ${ }^{1}$, Ioanna Koniari ${ }^{2}$, George D. Soufras ${ }^{3}$, Grigorios Tsigkas ${ }^{1}$, \\ Emmanouil Chourdakis ${ }^{4}$, Stefanos Despotopoulos ${ }^{1}$, Periklis Davlouros ${ }^{1}$, George Hahalis ${ }^{1}$ \\ ${ }^{1}$ Department of Cardiology, University of Patras Medical School, Rion, Patras, Achaia, Greece \\ ${ }^{2}$ Department of Electrophysiology, Queen Elizabeth Hospital, Birmingham, England \\ ${ }^{3}$ Department of Cardiology, "Saint Andrews" State General Hospital, Patras, Achaia, Greece \\ ${ }^{4}$ Krankenhaus der Barmherzigen Brüder, Trier, Germany
}

In an interesting report published in the "Cardiology Journal” concerning 95 patients treated for myocardial infarction with non-obstructive coronary arteries (MINOCA), López Pais et al. [1] found that when non-takotsubo MINOCA patients were evaluated the difference regarding psychiatric illnesses was $29.7 \%$ vs. $12.9 \%, \mathrm{p}=0.001$ compared to myocardial infarction (MI) with obstructive coronary arteries. Furthermore, in $11(11.6 \%)$ of these patients, pathophysiological mechanisms of MINOCA remained unknown.

In another recent report, involving 998 patients with angiografically proven $\mathrm{MI}$ in the real world, $82(8.2 \%)$ had a MINOCA and $40 \%$ were women. No evident etiology was detected in over $70 \%$ of MINOCA [2].

Therefore, it seems possible that new, yet unknown, pathophysiological mechanisms are involved in the pathogenesis of this conundrum.

So far, several other causes and pathogenetic mechanisms have been reported to be associated with this syndrome including coronary artery spasm, coronary artery dissection, coronary embolism, arrhythmias, mild plaque disruption, hypercoagulable status, type $2 \mathrm{MI}$, amyloid lightchain AL amyloidosis and clinically unrecognized myocarditis or takotsubo cardiomyopathy [2].

The prevalence of MINOCA among the MI patients ranged between $5 \%$ and $25 \%$ according to the registries [3]. In a recent report involving 199,163 MI admissions, 9092 consecutive unique patients had MINOCA, 2147 of them experienced a new major adverse cardiovascular event (MACE) and $1254(14 \%)$ of the patients died during mean follow-up of 4.5 years [4]. In this report, even after adjustment, low levels of total cholesterol were significantly associated with the composite endpoint of MACE as well as with long-term mortality. The authors had wondered about these results because hypercholesterolemia is considered as a causal factor for coronary artery disease and that the lowering of total cholesterol and low-density lipoprotein reduces cardiovascular risk in both primary and secondary prevention settings.

Indeed, it is being considered why, the Kounis hypersensitivity-associated type I variant coronary spasm which represents a manifestation of endothelial dysfunction or microvascular angina has not been included in this MINOCA report [1] as well as in others [5]. This variant includes patients with normal or nearly normal coronary arteries without predisposing factors for coronary artery disease and represents the most common type $(72.6 \%)$ of Kounis syndrome. In this variant, the acute release of inflammatory mediators may induce either coronary artery spasm without increased cardiac enzymes and troponins or coronary artery spasm progressing to acute MI with raised cardiac enzymes and troponins. The ensuing acute MI could lead to MACE including cardiogenic shock (2.3\%), cardiac arrest (6.3\%), death $5(2.9 \%)$ due to ventricular fibrillation,

Address for correspondence: Nicholas G. Kounis, MD, Queen Olgas Square, 7 Aratou Street, Patras 26221, Greece, e-mail: ngkounis@otenet.gr 
anterior ST-segment elevation MI and inferior ST-segment elevation MI [6].

The hypersensitivity-related Kounis syndrome inclusion in the MINOCA heterogenic group could well explain this cholesterol paradox observed in the aforementioned study [4]. Indeed, children and adolescents with clinical and laboratory manifestations of atopy have shown lower cholesterol concentrations throughout infancy, childhood and adolescence as compared with nonatopic subjects [7].

Kounis syndrome is rarely diagnosed but is not a rare disease. Indeed, the annual incidence of Kounis syndrome at the emergency department among all admissions and patients with allergy was 19.4 per 100,000 (27 of 138,911 ) and $3.4 \%$ (27 of 793), respectively [8]. In a recent report from Japan [9] the annual incidence of Kounis syndrome at emergency departments of Numazu City Hospital, Shizuoka, from 2012 to 2017 in patients with anaphylaxis, was $2 \%$ (2 of 100) and of Shizuoka Hospital, Juntendo University, from 2013 to 2017, was $2.2 \%$ (3 of 138), respectively. It is concluded herein that Kounis syndrome should always be excluded when physicians treat patients with allergy.

Specific biomarkers should be measured in cases of a suspicion of Kounis syndrome in MINOCA patients. These should include measurements of serum tryptase, histamine, eosinophils, total immunoglobulins (IgEs), cardiac enzymes and cardiac troponins supplemented with electrocardiography, echocardiography, angiography and modern tools such as thallium-201 single-photon emission computed tomography and dynamic cardiac magnetic resonance imaging [10]. In cases of drug-induced MINOCA the following diagnostic tests including specific IgEs, radioallergosorbent testing, enzyme linked immunosorbent assay, fluoroenzyme immunoassay, drug provocation test, basophil activation test and skin prick tests should be always performed. When a diagnosis of Kounis syndrome is established, treating type I variant with corticosteroids, $\mathrm{H} 1$ and $\mathrm{H} 2$ antihistamines and calcium channel blockers and nitrates is indicated.

All of the above show that MINOCA disease is neither benign nor a rare clinical entity. Searching, therefore, for its etiology and treatment that will provide a way for improving prognosis is of paramount importance but it is not an easy task. It is suggested herein that every MINOCA patient should undergo thorough clinical and laboratory diagnostic investigations in order to identify its causality and pathophysiological mechanisms. Future studies are necessary in order to dissolve the MINOCA conundrum.

\section{Conflict of interest: None declared}

\section{References}

1. Lopez Pais JL, Izquierdo Coronel B, Galán Gil D, et al. Psychoemotional disorders as incoming risk factors for myocardial infarction with non-obstructive coronary arteries. Cardiol J. 2018; 25(1): 24-31, doi: 10.5603/CJ.a2017.0139, indexed in Pubmed: 29240964.

2. Raparelli V, Elharram M, Shimony A, et al. Myocardial infarction with no obstructive coronary artery disease: angiographic and clinical insights in patients with premature presentation. Can J Cardiol. 2018; 34(4): 468-476, doi: 10.1016/j.cjca.2018.01.004, indexed in Pubmed: 29571427.

3. Montone RA, Niccoli G, Fracassi F, et al. Patients with acute myocardial infarction and non-obstructive coronary arteries: safety and prognostic relevance of invasive coronary provocative tests. Eur Heart J. 2018; 39(2): 91-98, doi: 10.1093/eurheartj/ ehx667, indexed in Pubmed: 29228159.

4. Nordenskjöld AM, Baron T, Eggers KM, et al. Predictors of adverse outcome in patients with myocardial infarction with nonobstructive coronary artery (MINOCA) disease. Int J Cardiol. 2018; 261: 18-23, doi: 10.1016/j.ijcard.2018.03.056, indexed in Pubmed: 29563017.

5. Agewall S, Beltrame JF, Reynolds HR, et al. WG on Cardiovascular Pharmacotherapy. ESC working group position paper on myocardial infarction with non-obstructive coronary arteries. Eur Heart J. 2017; 38(3): 143-153, doi: 10.1093/eurheartj/ehw149, indexed in Pubmed: 28158518.

6. Abdelghany M, Shah S, Subedi R, et al. Kounis syndrome: A review article on epidemiology, diagnostic findings, management and complications of allergic acute coronary syndrome. Int J Cardiol. 2017; 232: 1-4, doi: 10.1016/j.ijcard.2017.01.124, indexed in Pubmed: 28153536.

7. Pesonen M, Ranki A, Siimes MA, et al. Serum cholesterol level in infancy is inversely associated with subsequent allergy in children and adolescents. A 20-year follow-up study. Clin Exp Allergy. 2008; 38(1): 178-184, doi: 10.1111/j.1365-2222.2007.02875.x, indexed in Pubmed: 18028461.

8. Akoz A, Tanboga HI, Emet M, et al. prospective study of Kounis syndrome: clinical experience and cardiac magnetic resonance imaging findings for 21 patients. Acta Med Mediterraea. 2013; 9: 811-816.

9. Yanagawa $Y$, Kondo A, Ishikawa K, et al. Kounis syndrome should be excluded when physicians treat patients with anaphylaxis. Ann Allergy Asthma Immunol. 2017; 119(4): 392, doi: 10.1016/j. anai.2017.08.003, indexed in Pubmed: 28958381.

10. Kounis NG. Kounis syndrome: an update on epidemiology, pathogenesis, diagnosis and therapeutic management. Clin Chem Lab Med. 2016; 54(10): 1545-1559, doi: 10.1515/cclm-2016-0010, indexed in Pubmed: 26966931. 